\title{
Explaining the Gender Gap in
}

\section{COVID-19 Vaccination Attitudes}

\author{
Dimiter Toshkov ${ }^{1}$ \\ Institute of Public Administration, Leiden University, the Hague, the Netherlands
}

This version: 8 February 2022

(preprint, not peer reviewed)

${ }^{1}$ Corresponding author: Turfmarkt 99, 2511 DP, the Hague, the Netherlands, tel. +31715276771 , e-mail: d.d.toshkov@fgga.leidenuniv.nl. ORCID:0000-0002-7444-9340. 


\section{Abstract \\ Background}

Women have been significantly more likely than men to express hesitancy towards COVID-19 vaccination and, to a lesser extent, to refuse vaccination altogether. This gender gap is puzzling because women have been more likely to perceive higher risks from COVID-19, to approve more restrictive measures to fight the pandemic and to be more compliant with such measures.

\section{Methods}

This article studies the gender gap in COVID-19 vaccination attitudes using two nationally representative surveys of public opinion fielded in February 2021 and May 2021 in 27 European countries. The data is analyzed using generalized additive models and multivariate logistic regression. Results

The data analyses show that hypotheses about (a) pregnancy, fertility and breastfeeding concerns, (b) higher trust in Internet and social networks as sources of medical information, (c) lower trust in health authorities and (d) lower perceived risks of getting infected with COVID-19 cannot account for the gender gap in vaccine hesitancy. One explanation that receives support from the data is that women are more likely to believe that COVID-19 vaccines are unsafe and ineffective and this leads them to perceive the net benefits of vaccination as lower than the associated risks.

\section{Conclusions}

The gender gap in COVID-19 vaccine hesitancy results to a large extent from women perceiving higher risks than benefits of the vaccines. While accounting for this and other factors decreases the gap in vaccine hesitancy, it does not eliminate it completely, which suggests that further research is needed.

\section{Keywords}

Covid-19, gender, public opinion, risk perceptions, vaccine hesitancy 


\section{Introduction}

With the spread of the COVID-19 pandemic, significant differences between men and women emerged in relevant attitudes and behaviors. In particular, women were found to be less likely to declare intentions to get vaccinated against COVID-19, across very different national contexts [1-9], as confirmed by a recent systematic review and meta-analysis as well [10]. This is puzzling because it contrasts with the more cautious approach towards the pandemic exhibited by women, who were more likely to consider the pandemic a very serious health problem, to approve and to comply with restrictive measures imposed by governments to fight the pandemic [11]. Moreover, women are supposed to be more risk averse than men in general [12], which would lead us to think that they would choose the relative safety offered by vaccination against the well-known risks of the coronavirus and the associated disease. When it comes to vaccination more generally, there is conflicting evidence whether women are more likely than men to be vaccinated or to hold anti-vaccine attitudes [see overview in 10].

While higher levels of vaccine hesitancy for women have been noted in different samples across the world, there is relatively little work that tries to explain why this is the case and to account for the gender gap in vaccination attitudes [3]. The main objective of this article is to propose an explanation of this phenomenon by examining a number of hypotheses suggested by existing academic literature and popular discussions.

One possible explanation of the gender gap is concerns about how COVID-19 vaccines affect and interact with conception, pregnancy and breastfeeding. Health authorities and doctors in several countries (e.g. Bulgaria) have issued warnings about interference of the vaccination with these practices, which - warranted or not - might have influenced the attitudes of people [13,14]. Naturally, such concerns are likely to be higher among women, and younger women in particular. Relatedly, there is evidence for the effect of parenting role on vaccine hesitancy that is higher for women [15]. 
Another hypothesis refers to the alleged higher use of women of online social networks for health-related information[16]. Given that a lot of misinformation related to COVID-19 and vaccines has been spreading via online networks $[17,18]$, it is possible that this can account for the gender gap in vaccine hesitancy.

Relatedly, it has been proposed that women are less likely to trust official health authorities and the medical profession because of perceived gender bias in these institutions [19]. For many years women have been largely excluded from the profession, have had lower rates of participation in clinical trials and medical studies more generally, so that medical advice and recommended practices are often geared toward men and ill-adapted to women. Such generalized mistrust of health authorities can undermine support for the vaccines promoted by official health authorities.

The final hypothesis we consider refers to different risk attitudes and preferences of men and women [12,20]. This argument has two sides: perceived risks of COVID-19 and perceived risks and benefits of the vaccines. If women perceive lower risks of getting COVID-19 and lower severity and risk of death if they are infected by the virus (which would be justified based on clinical data [21,22]), this might result in higher vaccine hesitancy. But even if the COVID-19 risks are deemed high, different levels of hesitancy can appear from different assessments of the risks and benefits of vaccines, even if women and men are exposed to the same information. Yet, in 16 studies men showed lower perceived risk of getting COVID-19 and were not more concerned about the health consequences than women [23].

Still, for women the status quo before the vaccine development might be perceived as safer than vaccination based on hasty development of vaccines with unknown long-term consequences. There is some evidence that although women were not under-represented as participants in RCTs for vaccine effectiveness, there was insufficient reporting and analysis of COVID-19 vaccine data by gender and when disaggregated data was available, 'the majority of participants reporting adverse 
events were women.' [24,25] In an uncertain information environment surrounding the risks of vaccines, if men are more risk-accepting indeed, then this could account for their faster embrace of vaccines as a way to end the pandemic.

To test these hypotheses, the study uses data from two large comparative nationallyrepresentative surveys of public opinion fielded in the first half of 2021 in 27 European countries with a total of more than 50,000 respondents.

Understanding vaccine hesitancy and the gender gap in particular is important because these attitudes are strongly related to relevant behaviors, such as getting vaccinated or vaccinating one's children. Even small delays in the decision to vaccinate induced by vaccine hesitancy can have important consequences at the societal level for the efforts of governments and public health authorities to contain the spread of the virus and limit the impact of the disease. Identifying the reasons why women are more likely to express vaccine hesitancy can also help designing targeted information campaigns to combat the phenomenon.

\section{Methods}

This study is based on the analysis of two large comparative surveys of public opinion. The first survey (Standard Eurobarometer 94.3) covered 37 countries (from which we use the data on the $27 \mathrm{EU}$ member states for comparability purposes with the second survey that we anlyze) and was fielded at the request of the European Commission by Kantar [26]. The sample size per country was between 1,000 and 1,100 respondents (median of 1,040), with the exception of Luxembourg and Malta (which had between 500 and 600 respondents) and Germany (which had 1,575). Data collection took place between 12 February 2021 and 18 March 2021. Multi-stage, random sampling procures were used, with the first-stage sampling points being administrative regional units. In principle, interviews took place in person using CAPI (Computer Assistance Personal Interviewing), but because of the 
coronavirus pandemic, in 15 countries the interviews were conducted online and in 5 more both faceto-face and online interviews were used. In most countries, online sampling was also probabilistic (based on a Random Digit Dialing design, the country telephone directories or proprietary panels).

The second survey (Flash Eurobarometer 494) covered the 27 member states of the EU and was fielded in May 2021 at the request of the European Commission by Ipsos European Public Affairs, Brussels. Sampling was based on quota-based nationally-representative samples [27]. The interviews were web-based and conducted via self-administered questionnaires. The sample size for most countries was around 1,000 respondents (only Malta, Cyprus and Luxembourg had a smaller number of respondents at around 515 each). Both surveys provide post-stratification weights based on age, region and degree of urbanisation.

The main outcome variables of interest are COVID-19 vaccine hesitancy [28] and vaccine refusal. Respondents are considered 'vaccine hesitant' if they responded that they will 'Never' get vaccinated against COVID-19 or will get vaccinated 'Later' (but not 'some time in 2021') or 'Don't know' or prefer not to answer the question. (Excluding non-responses does not affect the results significantly). Vaccine refusal refers only to those who declare that they will 'Never' get vaccinated. The original formulation of the survey question was: 'When would you like to get vaccinated against COVID-19 (coronavirus)?' and the remaining answer categories, in addition to the ones mentioned above, are 'As soon as possible', 'Some time in 2021', and 'I have already been vaccinated'. For the operationalization and measurement of the covariates used in the analyses, see the Online supplementary material.

\section{Results}

In February 2021, 36.1\% of women expressed vaccine hesitancy versus $30.8 \%$ in the sample of respondents from the EU-27 (Pearson's chi-squared $\chi^{2}$ test statistic 86.19; p-value<0.001). In May 
$2021,28.7 \%$ of women versus $23.5 \%$ of men expressed vaccine hesitancy $(\chi 2=89.47$; p-value $<0.001)$. Therefore, while vaccine hesitancy declined significantly in the first part of 2021, the gender gap remained. The gender gaps with respect to vaccine refusal were smaller $-13.8 \%$ vs. $11.6 \%$ in February and $12.0 \%$ vs. $10.5 \%$ in May - but the differences between women and men were still significant $\left(\chi^{2}=29.96 ; \mathrm{p}\right.$-value $<0.001$ and $\chi^{2}=15.35 ; \mathrm{p}$-value $<0.001$, respectively $)$. As further evidence to the systematic nature of the gender gap, women were more likely to be vaccine hesitant than men in 22 out of 27 countries in February 2021 (the exceptions were Slovakia, Italy, Austria, Sweden and Bulgaria) and in 26 out of 27 countries in May 2021 (Ireland was the only exception). In four countries - Slovenia, Luxembourg, Cyprus and Bulgaria - the gap was 10 percentage points or wider. Figure 1 shows the distribution of vaccine hesitancy per gender, for each country and for the two time periods.

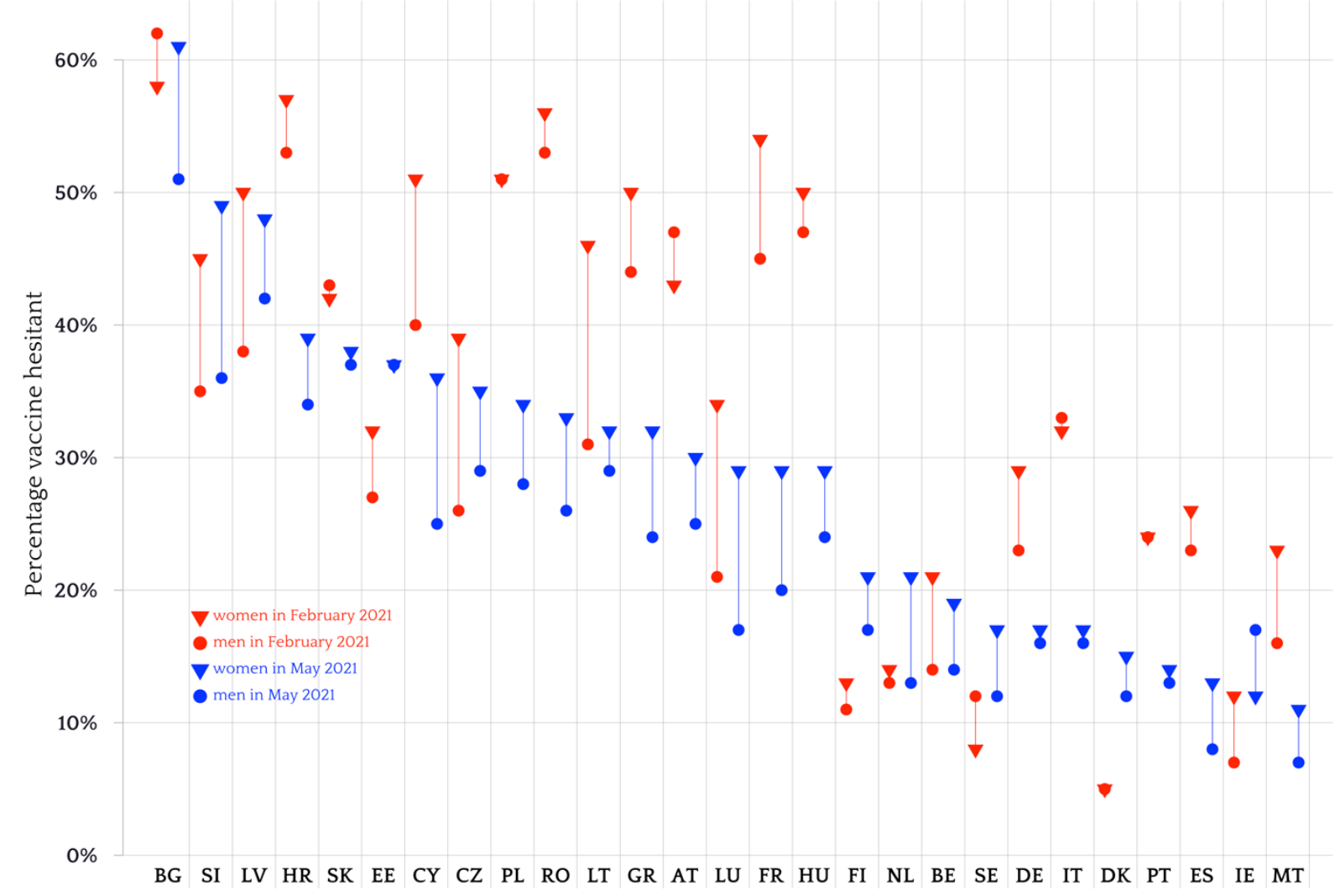

Figure 1. Percentage of women (triangles) and men (dots) who express COVID-19 vaccine hesitancy in February 2021 (in red) and in May 2021 (in blue) in each of the 27 member states of the EU. 
The first hypothesis we consider is that the gender gap results from concerns related to conception, pregnancy and breastfeeding. If such concerns are a major reason for the vaccine hesitancy gap, we would expect that the gap disappears as women pass fertility age. Figure 2 shows the results of non-parametric estimation of the effect of age on vaccine hesitancy in May 2021. The probabilities of vaccine hesitancy and refusal are derived from binomial generalized additive models with logic link [29], estimated separately for each gender, with age entered as a smooth term.
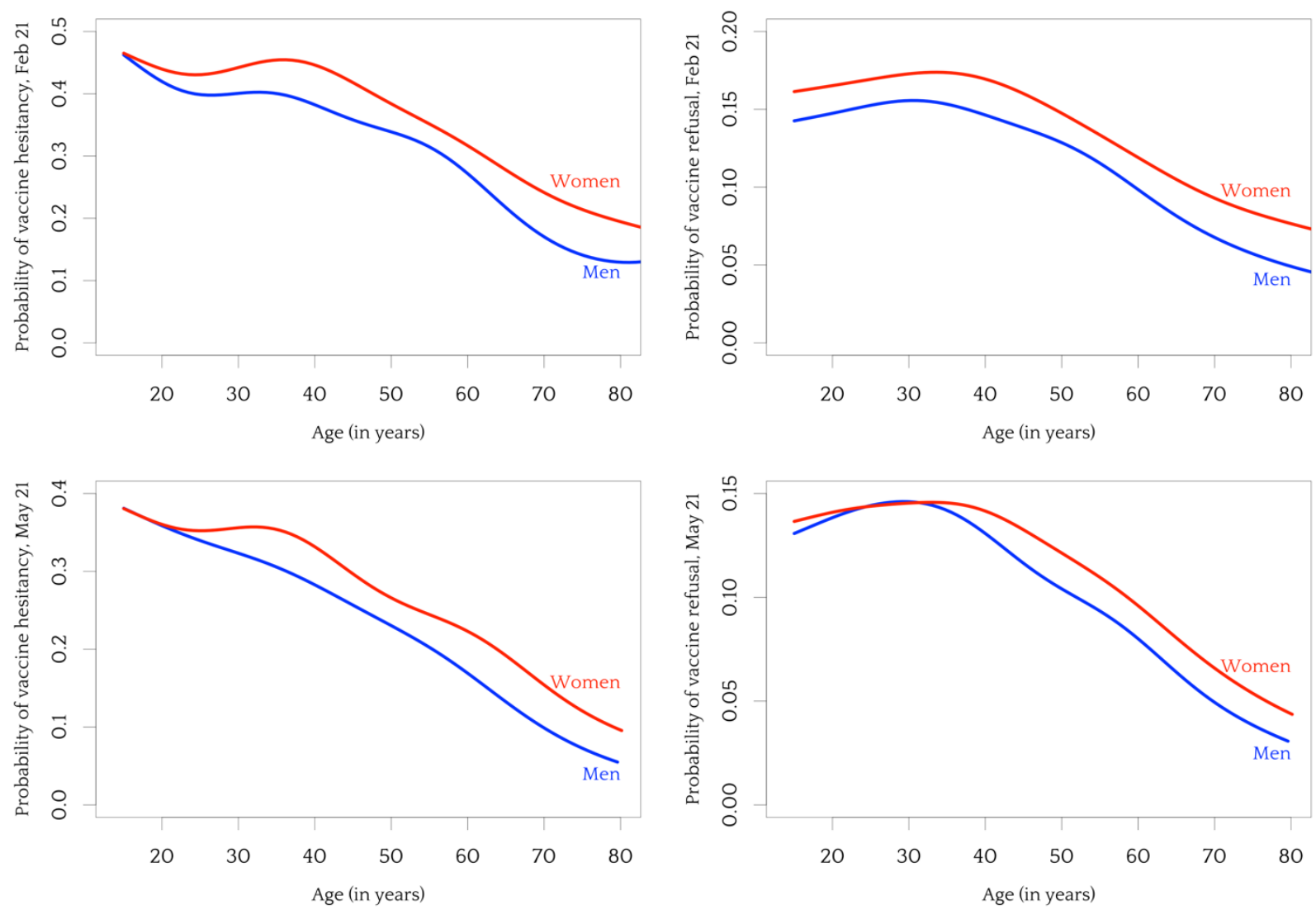

Figure 2. Probability of COVID-19 vaccine hesitancy (left) and vaccine refusal (right) as a function of age, per gender. Top row shows data from February 2021, bottom row from May 2021. Estimates based on binomial generalized additive models with logit link and smooth term for age.

As we can see from the plots in Figure 2, there is no evidence that the gender gap disappears or even declines as women pass fertility age. Very young women and men differ less in their probability of vaccine hesitancy and refusal (in May 2021), but, once it opens, the gender gap persists past the age 
when pregnancy and breastfeeding should be major personal concerns for women. While it is possible that once women develop concerns about a particular type of vaccine, such concerns could spill over into old age, this is less likely to be the case with COVID-19 vaccines, which have been developed very recently. The fact that the gender gap is evident as early as February 2021, before any reliable information about gender-specific side effects could have been present, also speaks against the idea that concrete gender-specific health concerns were responsible for the gap in attitudes.

Next, we consider the hypothesis that women have lower trust in health institutions and professionals due to a long history of male dominance in related professions and underrepresentation of women in medical studies. In February 2021, women were more likely to express trust in 'health and medical staff in their country: $83.9 \%$ vs. 82.7 for men $\left(\chi^{2}=6.64\right.$; $p$-value $\left.<0.01\right)$. Comparing the levels of trust women and men put into different sources of 'reliable information on COVID-19 vaccines' in particular, we find only minor differences in May 2021. Women trusted health authorities at 43.4\%, while men did at $44.2 \%\left(\chi^{2}=1.78 ; \mathrm{p}\right.$-value $\left.=0.18\right)$. Women trusted doctors and other medical professionals (for COVID-19 vaccine information) at 62\%, which was actually higher than the level for men $(59 \%)\left(\chi^{2}=24.97\right.$; p-value $\left.<0.001\right)$. It is remarkable that trust in health authorities $(53.8 \%$ women; $53.3 \%$ men) and medical professionals (74.5\% women; $69.8 \%$ men) as sources of trustworthy COVID-19 vaccine information declined between February and May 2021, just as vaccine acceptance increased during the same period.

Next, we consider the hypothesis that the gender gap results from higher trust of women in social networks and the Internet as sources of medical information. In February 2021, women reported higher general trust in online social networks $\left(22.0 \%\right.$ vs. $20.4 \%$ for men, $\chi 2==10.46$; $\mathrm{p}^{-}$ value $<0.01)$, but not in the Internet as a media $\left(40.0 \%\right.$ vs. $40.3 \%, \chi^{2}==0.18$; $p$-value $\left.=0.67\right)$. In May 2021, women were in fact less likely to report that they trust the Internet ( $7.3 \%$ vs $8.7 \%$ for men) and online social networks (5.3\% vs. 6.1\% for men) as sources of reliable information on COVID-19 
vaccines, in particular. They were, however, more likely to trust 'people around you (colleagues, friends and family)' $\left(15.9 \%\right.$ vs. $15.0 \%$ for men; $\chi^{2}==4.62$; $\mathrm{p}$-value $\left.=0.03\right)$, which is a significant predictor of vaccine hesitancy. Overall, there is no evidence that women are more likely to derive trustworthy information from the Internet and online networks for vaccine-relevant information, which is not consistent with the expectations of the hypothesis we consider.

Next, we turn to the hypothesis that the gender gap can be explained by reference to the different risks and benefits women perceive from COVID-19 and from the vaccines. Women were actually more likely to fear getting infected with COVID-19 in the future (in May 2021): women $45.6 \%$, men $40.6 \%, \chi^{2}=63.72 ; \mathrm{p}$-value $<0.001$. Furthermore, women were not more likely to believe that one can avoid COVID-19 without vaccination: women $47.4 \%$, men $48.2 \%, \chi^{2}=1.63$; pvalue $=0.20$. Hence, we have no evidence to conclude that women perceived lower risks of getting infected with COVID-19 [in line with 23], although we have no data as to how grave they thought the consequences of getting infected would be.

With regard to beliefs about the risks and benefits of vaccines, there are clear gender differences. Women were significantly more likely to agree that COVID-19 vaccines were developed too quickly to be safe (in February 2021, women 56.4\%, men 50.4\%, $\chi 2=101.02$; p-value $<0.001$; levels of agreement with this statement increased in May 2021, but the gender gap remained just as large). Similarly, women were significantly more likely to agree that COVID-19 vaccines could have unknown long-term side effects (in February 2021, women 70.3\%, men 63.8\%, $\chi 2=132.02$; p-value $<0.001$; levels were slightly lower in May 2021, but the gender gap increased slightly to 8 percentage points). They were also less likely to agree that a vaccine is the only way to stop the pandemic (in February 2021, women $69.7 \%$, men $72.4 \%, \chi^{2}=24.24 ; \mathrm{p}$-value $<0.001$; the gap increased slightly in May 2021).

When asked about vaccines in general, women expressed lower levels of agreement that vaccines are safe (women 75.0\%, men 80.0\%, $\chi 2==97.27$; p-value $<0.001$ ) and effective (women 
$83.0 \%$, men $85.1 \%, \chi^{2}==22.84 ; \mathrm{p}$-value $\left.<0.001\right)$. With respect to the belief that 'vaccines authorised in the European Union are safe', the gender gap was even wider (women $65.7 \%$, men $72.5 \%$, $\chi^{2}=143.35 ; \mathrm{p}$-value $\left.<0.001\right)$. In line with this pattern, women were less likely to agree that 'all in all, benefits of COVID-19 vaccines outweigh possible risks' (women 70.1\%, men 76.2\%, $\chi 2=122.02$; pvalue $<0.001$ ). To sum up the most significant differences between genders that we observe are with regard to beliefs about the risks and benefits of vaccines, with women being much more likely to express concern and consider that the risks outweigh the potential benefits.

These results are consistent with the hypothesis that differential risk perceptions account for the gender gap in COVID-19 vaccination attitudes, although with the important qualification that women perceive risks from COVID-19 as higher as well; just the risks of vaccines are perceived much higher than among men. But are the significant differences in attitudes and beliefs that we find enough to erase the gender gap? That is, are they enough to account for differences between men and women in full? To answer this question, we build regression models of vaccine hesitancy as a function of gender, and we add the potentially relevant variables discussed above to see whether the effect of gender is reduced in size or altogether disappears.

Table 1 shows the results from five logistic regression models (with logit link): Model 1 has only the gender variable as a predictor and serves as a baseline. Model 2 adds demographic variables and country indicators (the 26 country coefficients per model are not printed as they are not of substantive interest); Model 3 adds trust in various sources of COVID-19 information; Model 4 adds COVID-19 and vaccine-related beliefs; Model 5 adds only one additional variable: agreement that vaccines have more benefits than risks. The coefficients reported in the table are unexponentiated (the table shows the log of the odds ratios), so that a negative sign implies lower probability of vaccine hesitancy and a positive sign implies a higher probability of vaccine hesitancy. 
Table 1. Logistic regression models of vaccine hesitancy (Data: Eurobarometer from May 2021)

\begin{tabular}{|c|c|c|c|c|c|c|c|c|c|c|}
\hline \multirow[b]{2}{*}{ Variable } & \multicolumn{2}{|c|}{ Model 1} & \multicolumn{2}{|c|}{ Model 2} & \multicolumn{2}{|c|}{ Model 3} & \multicolumn{2}{|c|}{ Model 4} & \multicolumn{2}{|c|}{ Model 5} \\
\hline & $\log (\mathbf{O R})^{t}$ & $\mathrm{p}$-value & $\log (\mathbf{O R})^{\prime}$ & p-value & $\log (\mathbf{O R})^{t}$ & $\mathrm{p}$-value & $\log (\mathbf{O R})^{\prime}$ & $\mathrm{p}$-value & $\log (\mathbf{O R})^{1}$ & $\mathrm{p}$-value \\
\hline Gender [male] & -0.27 & $<0.001$ & -0.29 & $<0.001$ & -0.26 & $<0.001$ & -0.24 & $<0.001$ & -0.15 & 0.002 \\
\hline Age & & & -0.03 & $<0.001$ & -0.03 & $<0.001$ & -0.03 & $<0.001$ & -0.02 & $<0.001$ \\
\hline Education & & & -0.03 & $<0.001$ & -0.02 & $<0.001$ & -0.01 & 0.20 & -0.01 & 0.10 \\
\hline
\end{tabular}

Occupation

\begin{tabular}{|c|c|c|c|c|c|c|c|c|}
\hline Employee & - & & - & & - & & - & \\
\hline Manual worker & 0.53 & $<0.001$ & 0.42 & $<0.001$ & 0.35 & $<0.001$ & 0.28 & 0.003 \\
\hline No activity & 0.24 & $<0.001$ & 0.29 & $<0.001$ & 0.33 & $<0.001$ & 0.32 & $<0.001$ \\
\hline Self-employed & 0.29 & $<0.001$ & 0.21 & $<0.001$ & 0.14 & 0.044 & 0.14 & 0.050 \\
\hline Residence [city] & -0.22 & $<0.001$ & -0.16 & $<0.001$ & -0.14 & 0.003 & -0.13 & 0.008 \\
\hline Trust.EU.info & & & -0.95 & $<0.001$ & -0.63 & $<0.001$ & -0.49 & $<0.001$ \\
\hline Trust.gov.info & & & -0.63 & $<0.001$ & -0.32 & $<0.001$ & -0.17 & 0.033 \\
\hline Trust.health.info & & & -1.1 & $<0.001$ & -0.61 & $<0.001$ & -0.41 & $<0.001$ \\
\hline Trust.local.info & & & -0.43 & $<0.001$ & -0.40 & $<0.001$ & -0.38 & $<0.001$ \\
\hline Trust.doctors.info & & & -0.86 & $<0.001$ & -0.43 & $<0.001$ & -0.33 & $<0.001$ \\
\hline Trust.media.info & & & -0.39 & $<0.001$ & -0.14 & 0.090 & -0.08 & 0.35 \\
\hline Trust.web.info & & & 0.29 & $<0.001$ & 0.16 & 0.039 & 0.10 & 0.26 \\
\hline Trust.networks.info & & & 0.46 & $<0.001$ & 0.24 & 0.010 & 0.17 & 0.078 \\
\hline Trust.people.info & & & 0.05 & 0.30 & -0.07 & 0.21 & 0.00 & 0.96 \\
\hline Vaccines are safe & & & & & -0.86 & $<0.001$ & -0.52 & $<0.001$ \\
\hline Vaccines effective & & & & & -1.1 & $<0.001$ & -0.82 & $<0.001$ \\
\hline $\begin{array}{l}\text { Fears COVID-19 } \\
\text { infection }\end{array}$ & & & & & -0.90 & $<0.001$ & -0.70 & $<0.001$ \\
\hline
\end{tabular}




\begin{tabular}{|c|c|c|c|c|c|c|c|}
\hline \multirow[b]{2}{*}{ Variable } & Model 1 & Model 2 & Model 3 & \multicolumn{2}{|c|}{ Model 4} & \multicolumn{2}{|c|}{ Model 5} \\
\hline & $\log (\mathbf{O R})^{\prime} \quad \mathrm{p}$-value & $\log (\mathbf{O R})^{1} \quad \mathrm{p}$-value & $\log (\mathbf{O R})^{\prime} \quad$ p-value & $\log (\mathbf{O R})^{1}$ & p-value & $\log (\mathbf{O R})^{\prime}$ & $\mathrm{p}$-value \\
\hline $\begin{array}{l}\text { COVID-19 can be } \\
\text { avoided }\end{array}$ & & & & 0.78 & $<0.001$ & 0.68 & $<0.001$ \\
\hline $\begin{array}{l}\text { Vaccines developed } \\
\text { too fast }\end{array}$ & & & & 0.92 & $<0.001$ & 0.79 & $<0.001$ \\
\hline $\begin{array}{l}\text { Vaccines unknown } \\
\text { side effects }\end{array}$ & & & & 0.80 & $<0.001$ & 0.80 & $<0.001$ \\
\hline $\begin{array}{l}\text { Vaccines more } \\
\text { benefits than risks }\end{array}$ & & & & & & -1.9 & $<0.001$ \\
\hline AIC & 29954 & 20012 & 17508 & 137 & 99 & 122 & 296 \\
\hline $\mathrm{N}$ observations & 26106 & 19944 & 19944 & 19 & 44 & 199 & 44 \\
\hline
\end{tabular}

According to the baseline Model 1, men are significantly less likely to be vaccine hesitant than women. Adding demographic variables even increases slightly the gender gap. Adding the trust-related variables does little to reduce the effect of gender, although many of these variables have significant effects as such. Even when we add relevant COVID-19 and vaccine-relevant beliefs, the coefficient of gender drops in size only from -0.27 to -0.24 , which makes for a small decrease in the size of the gender gap in vaccine hesitancy, conditional on all these variables. Remarkably, when a single additional variable is added in Model 5 - the belief that the benefits of vaccines outweigh the associated risks, the effect of gender drops significantly to -0.14 . The fit of Model 5 is also significantly better than the rest. We can conclude that although there are many significant predictors of vaccine hesitancy in Europe that are associated with gender, the belief that the benefits of vaccines outweigh the risks is by far the most important variable that can reduce the gender gap in vaccine hesitancy.

Including interactions of gender with the other variables does not reveal any heterogeneous effects for men and women. Mediation models in which gender can affect vaccine hesitancy both 
directly and via trust in different information sources and via relevant beliefs deliver evidence for significant direct and indirect effects as well.

When the same set of models are estimated using vaccine refusal as the outcome variable, the effect of gender is smaller to begin with, and disappears completely once we include the variables related to vaccine beliefs (see Table A1 in the Online supplementary materials). This suggests that the higher vaccine hesitancy among women is not due to fundamental opposition to vaccination, but to higher levels of caution, in line with the results reported above.

There are some significant differences in the reasons men and women endorse for and against vaccination. Women were less likely to agree that vaccination is a civic duty (women $52.9 \%$, men 61.3\%, $\chi^{2}=186.96 ; \mathrm{p}$-value $<0.001$ ) and to support compulsory vaccination (women 39.0\%, men $45.9 \%, \chi^{2}=126.28 ; \mathrm{p}$-value $\left.<0.001\right)$. Among the vaccine hesitant respondents, women are less likely to be against vaccine in general, to say that the infection risk is too low for them personally, to think that the pandemic will be over soon anyway, and - importantly - to agree that the risks of COVID-19 are exaggerated. However, women are more likely to be worried about side effects and to think that vaccines are not effective (see Figures A1 and A2 in the Online supplementary material). (Note that these questions were asked only to vaccine hesitant respondents, so they do not allow us to make inferences about the prevalence of these attitudes among all women and all men).

\section{Discussion}

Using data from two large comparative surveys of public opinion in 27 European countries from the first half of 2021, this article showed that a significant gender gap existed with respect to COVID-19 vaccine hesitancy and, to a lesser extent, vaccine refusal. The hypotheses that the gap results from (a) pregnancy, breastfeeding and fertility concerns, (b) higher trust in Internet and social networks as sources of medical information, (c) lower trust in health authorities and (d) lower perceived risks of 
getting infected with COVID-19 did not find support from the statistical tests and analyses conducted.

The one hypothesis that is consistent with the data is that women were more likely to perceive COVID-19 vaccines as unsafe and ineffective and this leads them to consider the net benefits of vaccination as lower than the associated risks. The last variable appears to be the most important factor behind the gender gap in COVID-19 vaccination attitudes.

The differential assessment of potential risks and benefits of the COVID-19 vaccine appeared quite early in 2021, before concrete and reliable information was available about possible side effects. This suggests that the differential assessment might be due to a form of 'status quo bias' that is more prevalent among women [30] and interacts with risk aversion.

The study has important limitations related to its temporal and geographical focus, which was limited to European countries only. But the evidence for a gender gap in vaccination attitudes from across the world suggests that the causal structure behind it might be similar as well to the one outlined in this article for the European case.

Both surveys analyzed in this article were fielded in the first half of 2021, when the mass vaccination campaigns were being rolled out in Europe and not much was known yet about the realworld effectiveness and possible side effects of the vaccines. It is possible that the gender gap and the reasons behind it changed with time, but our results still remain substantively relevant and important. Even if strong government incentives for COVID-19 vaccinations reduced vaccine hesitancy and closed the gender gap later in 2021 and 2022, related attitudes, such as those towards the vaccination of children or getting booster vaccination shots, likely have similar antecedents. In this respect the relevance of this study goes beyond the immediate empirical context of early-stage COVID-19 vaccination campaigns. One of the important open questions that remains is to what extent vaccine attitudes determine behavior and whether hesitancy can be overcome with targeted information 
campaigns, for example. But even if attitudes just delay vaccination, the public health consequences can be considerable.

In this respect, the results of this study suggest that information campaigns might need to be gender-specific. If women are more likely to assume that the risks of vaccination outweigh the benefits even in the absence of strong evidence to this effect, messages need to be crafted to address such concerns early on in public health campaigns. Some existing research, however, does not find strong gender-specific effects of information messages on the role of COVID-19 vaccination [3,7,31]. Yet, the fact that we do not find support for the ideas that the gender gap results from structural factors, such as systematically lower trust of health authorities or higher use of social networks as sources of medical information, implies that communication can be effective in closing the gender gap.

\section{Funding}

No specific funding was used for this research.

\section{Conflict of interest}

The author declares no conflict of interest.

\section{Key-points}

- Women were systematically and significantly more likely to express vaccine hesitant attitudes towards COVID-19 vaccines in Europe during the first part of 2021.

- The gender gap in vaccine hesitancy did not results from pregnancy and fertility concerns, higher trust in Internet and social networks as sources of medical information, lower trust in health authorities or lower perceived risks of getting infected with COVID-19.

- A major factor behind the gender gap in vaccine hesitancy was the higher relative risks versus benefits of COVID-19 vaccines perceived by women compared to men. 


\section{References}

1. Enticott J, Gill JS, Bacon SL, Lavoie KL, Epstein DS, Dawadi S, et al. Attitudes towards vaccines and intention to vaccinate against COVID-19: a cross-sectional analysis-implications for public health communications in Australia. BMJ Open. 2022 Jan 1;12(1):e057127.

2. Lindholt MF, Jørgensen F, Bor A, Petersen MB. Public acceptance of COVID-19 vaccines: cross-national evidence on levels and individual-level predictors using observational data. BMJ Open. 2021;11(6):e048172.

3. Galasso V, Profeta P, Foucault M, Pons V. COVID-19 Vaccine's Gender Paradox. medRxiv. 2021 Jan 1;2021.03.26.21254380.

4. Ishimaru T, Okawara M, Ando H, Hino A, Nagata T, Tateishi S, et al. Gender differences in the determinants of willingness to get the COVID-19 vaccine among the working-age population in Japan. Hum Vaccin Immunother. 2021 Nov 2;17(11):3975-81.

5. Solís Arce JS, Warren SS, Meriggi NF, Scacco A, McMurry N, Voors M, et al. COVID-19 vaccine acceptance and hesitancy in low- and middle-income countries. Nat Med. 2021;27(8):1385-94.

6. Karlsson LC, Soveri A, Lewandowsky S, Karlsson L, Karlsson H, Nolvi S, et al. Fearing the disease or the vaccine: The case of COVID-19. Pers Individ Dif. 2021;172:110590.

7. Kreps SE, Kriner DL. Factors influencing Covid-19 vaccine acceptance across subgroups in the United States: Evidence from a conjoint experiment. Vaccine. 2021;39(24):3250-8.

8. Nery Jr N, Ticona JPA, Cardoso CW, Prates APPB, Vieira HCA, Salvador de Almeida A, et al. COVID-19 vaccine hesitancy and associated factors according to sex: A population-based survey in Salvador, Brazil. PLoS One. 2022;17(1):e0262649.

9. Lazarus J V, Wyka K, Rauh L, Rabin K, Ratzan S, Gostin LO, et al. Hesitant or Not? The Association of Age, Gender, and Education with Potential Acceptance of a COVID-19 
Vaccine: A Country-level Analysis. J Health Commun. 2020 Oct 2;25(10):799-807.

10. Zintel S, Flock C, Arbogast AL, Forster A, von Wagner C, Sieverding M. Gender differences in the intention to get vaccinated against COVID-19: a systematic review and meta-analysis. J Public Health (Bangkok). 2022;

11. Galasso V, Pons V, Profeta P, Becher M, Brouard S, Foucault M. Gender differences in COVID-19 attitudes and behavior: Panel evidence from eight countries. Proc Natl Acad Sci. 2020 Nov 3;117(44):27285 LP - 27291.

12. Byrnes JP, Miller DC, Schafer WD. Gender differences in risk taking: A meta-analysis. Psychol Bull. 1999;125(3):367.

13. Galanis P, Vraka I, Siskou O, Konstantakopoulou O, Katsiroumpa A, Kaitelidou D. Predictors of COVID-19 vaccination uptake and reasons for decline of vaccination: a systematic review. medRxiv. 2021 Jan 1;2021.07.28.21261261.

14. Sutton D, D'Alton M, Zhang Y, Kahe K, Cepin A, Goffman D, et al. COVID-19 vaccine acceptance among pregnant, breastfeeding, and nonpregnant reproductive-aged women. Am J Obstet Gynecol MFM. 2021;3(5):100403.

15. Reich JA. "We are fierce, independent thinkers and intelligent": Social capital and stigma management among mothers who refuse vaccines. Soc Sci Med. 2020;257:112015.

16. Alvarez-Galvez J, Salinas-Perez JA, Montagni I, Salvador-Carulla L. The persistence of digital divides in the use of health information: a comparative study in 28 European countries. Int J Public Health. 2020;65(3):325-33.

17. Johnson NF, Velásquez N, Restrepo NJ, Leahy R, Gabriel N, El Oud S, et al. The online competition between pro-and anti-vaccination views. Nature. 2020;582(7811):230-3.

18. Wilson SL, Wiysonge C. Social media and vaccine hesitancy. BMJ Glob Heal. 2020;5(10):e004206. 
19. Corra M, Carter JS. Shadow of the Past?: Assessing Racial and Gender Differences in Confidence in the Institutions of Science and Medicine. Black Women, Gend + Fam. 2008 Feb 7;2(1):54-83.

20. Flynn J, Slovic P, Mertz CK. Gender, race, and perception of environmental health risks. Risk Anal. 1994;14(6):1101-8.

21. Márquez EJ, Trowbridge J, Kuchel GA, Banchereau J, Ucar D. The lethal sex gap: COVID19. Immun Ageing. 2020;17(1):1-8.

22. Wenham C, Smith J, Morgan R. COVID-19: the gendered impacts of the outbreak. Lancet. 2020;395(10227):846-8.

23. Lewis A, Duch R. Gender differences in perceived risk of COVID-19. Soc Sci Q. 2021 Sep 1;102(5):2124-33.

24. Vassallo A, Shajahan S, Harris K, Hallam L, Hockham C, Womersley K, et al. Sex and Gender in COVID-19 Vaccine Research: Substantial Evidence Gaps Remain. Front Glob women’s Heal. 2021 Nov 1;2:761511.

25. Green MS, Peer V, Magid A, Hagani N, Anis E, Nitzan D. Gender Differences in Adverse Events Following the Pfizer-BioNTech COVID-19 Vaccine. Vol. 10, Vaccines . 2022.

26. European Commission. Eurobarometer 94.3 (2021). GESIS Data Archive ZA7780. Brussels; 2021.

27. European Commission. Flash Eurobarometer 494 (Attitudes on Vaccination against Covid-19) GESIS Data Archive ZA7771. 2021.

28. MacDonald NE. Vaccine hesitancy: Definition, scope and determinants. Vaccine. 2015;33(34):4161-4.

29. Wood SN. Generalized additive models: an introduction with R. CRC press; 2017.

30. Bekir I, Doss F. Status quo bias and attitude towards risk: An experimental investigation. Manag 
Decis Econ. 2020 Jul 1;41(5):827-38.

31. Doğan İ, Baruh L, Cemalcilar Z, Kuru O, Yıldırım K, Çarkoğlu A. Biased perceptions against female scientists affect intentions to get vaccinated for COVID-19. Public Underst Sci. 2021 Dec 1;31(2):239-51. 


\section{Online supplementary material}

\section{Operationalization and measurement of the covariates used in the analysis}

The main demographic predictors of interest were age (measured in years), education (measured as the age at which full-time education was stopped), gender, place of residence ('Large town/city' vs. 'Small or medium-sized town' and 'A rural area of village'), and professional occupation (with four categories: 'Self-employed', 'Employee', 'Manual worker', and 'Without a professional activity').

Trust in authorities was measured with the question: 'How much trust do you have in certain institutions? For each of the following institutions, do you tend to trust it or tend not to trust it?' with respect to 'Health and medical staff in (OUR COUNTRY)'.

General trust in media was measured with the question: 'How much trust do you have in certain media? For each of the following media, do you tend to trust it or tend not to trust it?' with respect to 'The internet' and 'Online social networks'.

Trust in different authorities with respect to COVID-19-related information was measured via the question 'Among the following sources, which ones would you trust more to give you reliable information on COVID19 vaccines?', with multiple answers possible from the following categories: 'The European Union', 'The National Government', 'The National Health Authorities', 'The regional or local public authorities', 'Health professionals, doctors, nurses and pharmacists', 'Media (television, radio, newspapers', 'Websites', 'Online social networks', 'People around you (colleagues, friends and family)' and 'Don't know').

Several vaccine-related beliefs were measured: 'Vaccines are safe', 'Vaccines are effective', 'I believe vaccines authorised in the European Union are safe', 'All in all, benefits of COVID-19 vaccines outweigh possible risks', 'COVID-19 vaccines are being developed, tested and authorised too quickly to be safe', 'COVID-19 vaccines could have long term side-effects that we do not know yet', 'A vaccine is the only way to end the pandemic', 'You can avoid being infected by COVID-19 without being 
vaccinated', 'COVID-19 vaccination should be compulsory' and 'Everyone should get vaccinated against COVID-19, it is a civic duty'. Originally, answers to all these were recorded on 4-point agreement scales, which were collapsed in two categories ('Agree totally or tend to agree' vs. 'Disagree totally or tend to disagree'). An additional item related to knowledge about COVID-19 was used: whether the respondent fears to be infected with COVID-19 in the future [yes/no answer]. 
Table A1. Logistic regression models of vaccine refusal (Data: Eurobarometer, May 2021)

\begin{tabular}{|c|c|c|c|c|c|c|c|c|c|c|}
\hline \multirow[b]{2}{*}{ Variable } & \multicolumn{2}{|c|}{ Model 1} & \multicolumn{2}{|c|}{ Model 2} & \multicolumn{2}{|c|}{ Model 3} & \multicolumn{2}{|c|}{ Model 4} & \multicolumn{2}{|c|}{ Model 5} \\
\hline & $\log (\mathbf{O R})^{\prime}$ & $\mathrm{p}$-value & $\log (\mathbf{O R})^{\prime}$ & $\mathrm{p}$-value & $\log (\mathbf{O R})^{\prime}$ & $\mathrm{p}$-value & $\log (\mathbf{O R})^{\prime}$ & $\mathrm{p}$-value & $\log (\mathbf{O R})^{\prime}$ & $\mathrm{p}$-value \\
\hline Gender [male] & -0.15 & $<0.001$ & -0.16 & $<0.001$ & -0.10 & 0.046 & -0.06 & 0.27 & 0.05 & 0.44 \\
\hline Age & & & -0.02 & $<0.001$ & -0.02 & $<0.001$ & -0.02 & $<0.001$ & -0.02 & $<0.001$ \\
\hline Education & & & -0.03 & $<0.001$ & -0.01 & 0.046 & 0.00 & 0.56 & -0.01 & 0.32 \\
\hline \multicolumn{11}{|l|}{ Occupation } \\
\hline Employee & & & - & & - & & - & & - & \\
\hline Manual worker & & & 0.31 & 0.001 & 0.12 & 0.23 & 0.02 & 0.85 & -0.03 & 0.83 \\
\hline No activity & & & 0.21 & $<0.001$ & 0.24 & $<0.001$ & 0.24 & 0.001 & 0.25 & 0.001 \\
\hline Self-employed & & & 0.34 & $<0.001$ & 0.26 & $<0.001$ & 0.19 & 0.024 & 0.19 & 0.026 \\
\hline Residence [city] & & & -0.21 & $<0.001$ & -0.13 & 0.015 & -0.12 & 0.056 & -0.11 & 0.090 \\
\hline Trust.EU.info & & & & & -1.4 & $<0.001$ & -1.0 & $<0.001$ & -0.79 & $<0.001$ \\
\hline Trust.gov.info & & & & & -0.74 & $<0.001$ & -0.26 & 0.035 & -0.02 & 0.88 \\
\hline Trust.health.info & & & & & -1.4 & $<0.001$ & -0.92 & $<0.001$ & -0.68 & $<0.001$ \\
\hline Trust.local.info & & & & & -0.57 & $<0.001$ & -0.40 & 0.005 & -0.34 & 0.022 \\
\hline Trust.doctors.info & & & & & -1.2 & $<0.001$ & -0.75 & $<0.001$ & -0.67 & $<0.001$ \\
\hline Trust.media.info & & & & & -0.56 & $<0.001$ & -0.19 & 0.11 & -0.06 & 0.61 \\
\hline Trust.web.info & & & & & 0.30 & $<0.001$ & 0.18 & 0.064 & 0.12 & 0.22 \\
\hline Trust.networks.info & & & & & 0.51 & $<0.001$ & 0.39 & $<0.001$ & 0.35 & 0.002 \\
\hline Trust.people.info & & & & & -0.31 & $<0.001$ & -0.42 & $<0.001$ & -0.32 & $<0.001$ \\
\hline Vaccines are safe & & & & & & & -0.68 & $<0.001$ & -0.38 & $<0.001$ \\
\hline Vaccines effective & & & & & & & -0.82 & $<0.001$ & -0.64 & $<0.001$ \\
\hline $\begin{array}{l}\text { Fears COVID-19 } \\
\text { infection }\end{array}$ & & & & & & & -1.2 & $<0.001$ & -1.0 & $<0.001$ \\
\hline
\end{tabular}




\begin{tabular}{|c|c|c|c|c|c|c|c|}
\hline \multirow[b]{2}{*}{ Variable } & \multirow{2}{*}{$\begin{array}{l}\text { Model } 1 \\
\log (\text { OR })^{\prime} \quad \text { p-value }\end{array}$} & \multirow{2}{*}{$\begin{array}{l}\text { Model } 2 \\
\log (\text { OR })^{\prime} \quad \text {-value }\end{array}$} & \multirow{2}{*}{$\begin{array}{l}\text { Model } 3 \\
\log (\mathrm{OR})^{\prime} \quad \text {-value }\end{array}$} & \multicolumn{2}{|c|}{ Model 4} & \multicolumn{2}{|c|}{ Model 5} \\
\hline & & & & $\log (\mathbf{O R})^{\prime}$ & $\mathrm{p}$-value & $\log (\mathbf{O R})^{\prime}$ & $\mathrm{p}$-value \\
\hline $\begin{array}{l}\text { COVID-19 can be } \\
\text { avoided }\end{array}$ & & & & 0.91 & $<0.001$ & 0.79 & $<0.001$ \\
\hline $\begin{array}{l}\text { Vaccines developed } \\
\text { too fast }\end{array}$ & & & & 0.67 & $<0.001$ & 0.51 & $<0.001$ \\
\hline $\begin{array}{l}\text { Vaccines unknown } \\
\text { side effects }\end{array}$ & & & & 0.95 & $<0.001$ & 0.92 & $<0.001$ \\
\hline $\begin{array}{l}\text { Vaccines more } \\
\text { benefits than risks }\end{array}$ & & & & & & -1.7 & $<0.001$ \\
\hline
\end{tabular}




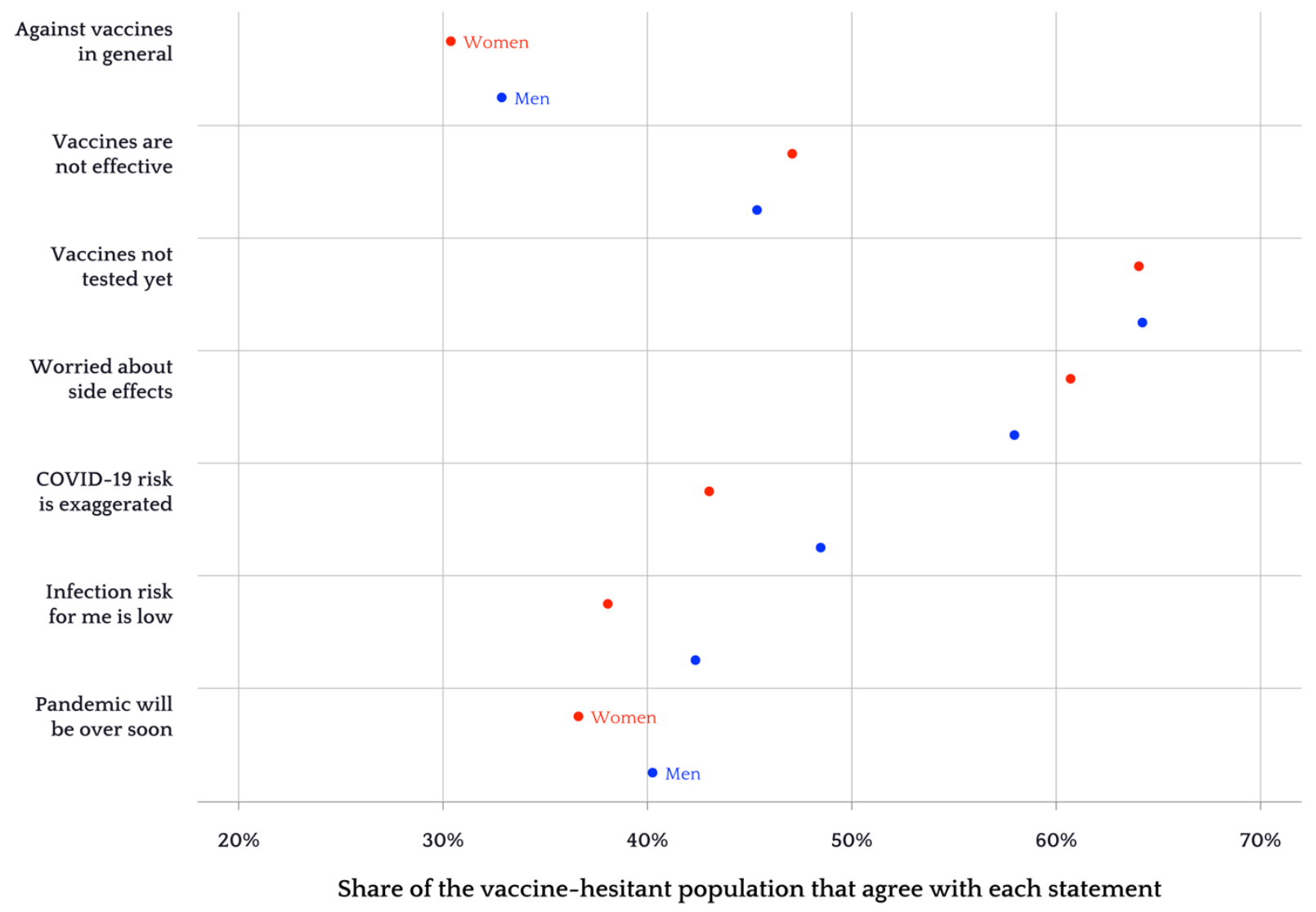

Figure A1. Percentage of women (red dots) and men (blue dots) who endorse a particular reason (shown on the y-axis) for COVID-19 vaccine hesitancy, from those who are vaccine hesitant, May 2021 


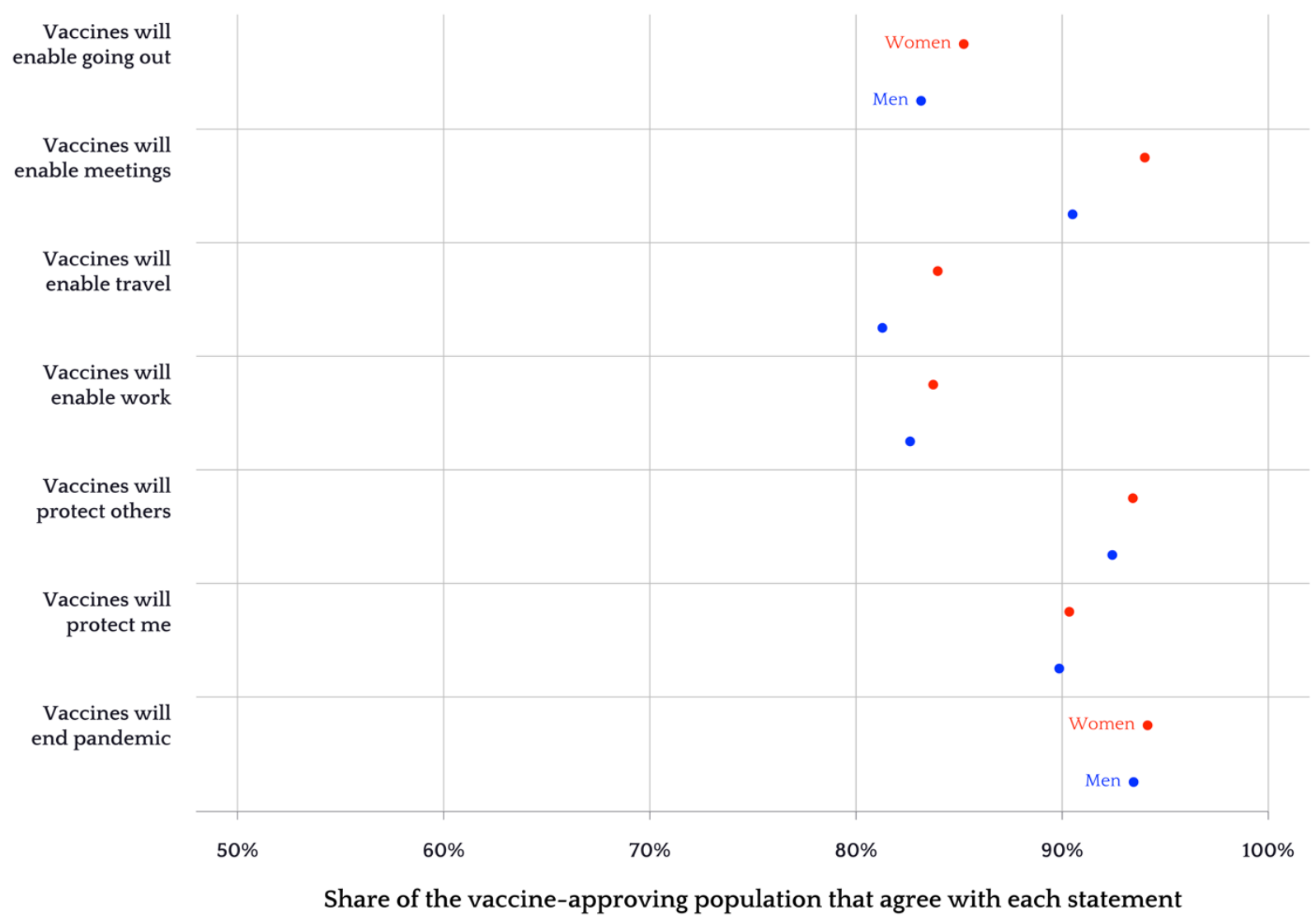

Figure A2. Percentage of women (red dots) and men (blue dots) who endorse a particular reason (shown on the y-axis) for COVID-19 vaccine acceptance, from those who are vaccine accepting, May 2021 